\title{
ABOUT DIRECT SUMMANDS OF PROJECTIVE MODULES OVER LAURENT POLYNOMIAL RINGS
}

\author{
SATYA MANDAL \\ (Communicated by Louis J. Ratliff, Jr.)
}

\begin{abstract}
Suppose $A$ is a local ring and $R=A\left[X, X^{-1}\right]$ is a Laurent polynomial ring. We prove that for projective $R$-modules $P$ and $Q$ with rank $Q<\operatorname{rank} P$, if $Q_{f}$ is a direct summand of $P_{f}$ for a doubly monic polynomial $f$ then $Q$ is also a direct summand of $P$. We also prove the analogue of the Horrock's theorem for Laurent polynomials rings.
\end{abstract}

\section{INTRODUCTION}

In [R] Roy proved that if $R=A[X]$ is a polynomial ring over a local ring $A$ and if $P$ and $Q$ are two projective $R$-modules with $\operatorname{rank} Q<\operatorname{rank} P$, then $Q_{f}$ is a direct summand of $P_{f}$ for some monic polynomial $f$, implies that $Q$ is a direct summand of $P$. (All rings are assumed to be noetherian and commutative, and the modules are assumed to be finitely generated.)

In this paper, we shall extend this result of Roy and the consequences to the Laurent polynomial ring case.

We need the following definition for the subsequent discussions.

(1.1) Definition. Suppose that $R=A\left[X, X^{-1}\right]$ is a Laurent polynomial ring over a commutative ring $A$. An element $f$ in $R$ is called a doubly monic Laurent polynomial if the coefficients of the highest degree and the lowest degree terms are units.

We shall be extending the above theorem of Roy [R] to the Laurent polynomial situation by replacing "monic polynomial" by "doubly monic Laurent polynomial" in his statement (see (2.1) below).

As a consequence, we shall be proving the Laurent polynomial version of the Horrock's theorem; that is, if $P$ is a projective module over a Laurent polynomial ring $R=A\left[X, X^{-1}\right]$ over a local commutative noetherian ring $A$ and if $P_{f}$ is free for some doubly monic Laurent polynomial $f$ then $P$ is also free.

Received by the editors November 17, 1989 and, in revised form, August 20, 1990.

1980 Mathematics Subject Classification (1985 Revision). Primary $13 \mathrm{C} 10$. Kansas.

Partially supported by the General Research Fund and New Faculty Grant of the University of 


\section{THE MAIN DISCUSSIONS}

The following is the main result of this paper:

(2.1) Theorem. Suppose that $R=A\left[X, X^{-1}\right]$ is a Laurent polynomial ring over a local noetherian commutative ring $A$. Also suppose that $P$ and $Q$ are two projective $R$-modules with $\operatorname{rank} Q<\operatorname{rank} P$. If $Q_{f}$ is a direct summand of $P_{f}$ for some doubly monic Laurent polynomial $f$, then $Q$ is also a direct summand of $P$.

Before we go into the proof of $(2.1)$, we discuss the consequences of this theorem.

(2.2) Theorem. Let $R=A\left[X, X^{-1}\right]$ be as above (2.1) and $P, Q$ be two projective $R$-modules such that $P_{f}$ is isomorphic to $Q_{f}$ for some doubly monic Laurent polynomial $f$. Then,

(i) $Q$ is a direct summand of $P \oplus L$ for all nonzero projective $R$-modules $L$.

(ii) $P$ is isomorphic to $Q$ if $P$ or $Q$ has a rank one direct summand.

(iii) $P \oplus L$ is isomorphic to $Q \oplus L$ for all rank one projective $R$-modules $L$.

(iv) $P$ and $Q$ have same number of generators.

Proof of (2.2). We shall only prove (ii), because the rest of the arguments are as in Roy's proof [R, Proposition 3.1].

To Prove (ii), let $P=P^{\prime} \oplus L$ for some rank one projective $R$-module $L$. By (2.1) $Q \approx P^{\prime} \oplus L^{\prime}$ for some rank one projective $R$-module $L^{\prime}$. Now it is enough to prove that $L \approx L^{\prime}$.

Since $P_{f} \approx Q_{f}$, it follows that $L_{f} \approx L_{f}^{\prime}$. Hence $\left(L^{\prime} L^{-1}\right)_{f}$ is free. This means that $L^{\prime} L^{-1}$ is isomorphic to an invertible ideal $I$ of $R=A\left[X, X^{-1}\right]$ such that $I$ contains some power of $f$. Write $J=I \cap A[X]$. As $I$ contains a doubly monic Laurent polynomial, $J$ is also an invertible ideal in $A[X]$. Moreover, since $J$ also contains a monic polynomial, $J$ is a principal ideal. (See [NN] for an elementary proof of this fact.) Now it follows that $I$ is a principal ideal and hence $L \approx L^{\prime}$. The proof of (ii) is complete.

The following Laurent polynomial analogue of Horrock's theorem is an immediate sequence of (2.2).

(2.3) Corollary. Suppose $R=A\left[X, X^{-1}\right]$ is as in (2.1) and $P$ is a projective $R$-module. If $P_{f}$ is free for some doubly monic Laurent polynomial, then $P$ is free.

(2.4) Remark. The nonlocal version of (2.3) is also true; that is, if $R=$ $A\left[X, X^{-1}\right]$ is a Laurent polynomial ring over a (nonlocal) noetherian commutative ring $A$ and if $P$ is a projective $R$-module with $P_{f}$ free for some doubly monic Laurent polynomial $f$, then $P$ is free. (See [BR, Remark (3.6)] for a proof in the rank one case.) 
Proof. Without loss of generality, we can assume that $f$ is a monic polynomial in $A[X]$ with $f(0)=1$. Now $X A[X]+f A[X]=A[X]$. Since $P_{f}$ is free, by patching $P$ with a free $A[X]_{f}$-module, we can get a projective $A[X]$-module $Q$ with $Q_{X}=P$. Again, by construction $Q_{f}$ is free. By the Quillen-Suslin theorem it follows that $Q$ is free and hence, $P$ is also free.

\section{Proof of Theorem (2.1)}

In this section, we shall prove Theorem (2.1). The proof is in the line of Roy's proof $[R]$ with necessary modification for Laurent polynomial rings.

First we have to prove a few lemmas.

(3.1) Lemma. Suppose $R$ is a noetherian commutative ring and $P, Q$ are two projective $R$-modules. Suppose $\phi: Q \rightarrow P$ is an $R$-linear map. For an ideal $I$ of $R$, if $\phi$ is a split monomorphism modulo $I$ then $\phi_{1+I}: Q_{1+I} \rightarrow P_{1+I}$ is also a split monomorphism.

Proof. We need to check that $\phi$ is a split monomorphism after localizing at maximal ideals containing $I$. So we can assume that $R$ is local. In this case, it is obvious.

(3.2) Lemma. Suppose $R=A\left[X, X^{-1}\right]$ is a Laurent polynomial ring over a local noetherian commutative ring $A$ with maximal ideal $\mathbf{m}$. Let $P$ and $Q$ be two projective $R$-modules and $\phi: Q \rightarrow P$ be an $R$-linear map. If $\phi$ is a split monomorphism modulo $\mathbf{m}$ and if $\phi_{f}$ is a split monomorphism for some doubly monic Laurent polynomial $f$, then $\phi$ is also a split monomorphism.

Proof. By (3.1) $\phi_{1+\mathrm{m} R}$ is a split monomorphism. So, there is an element $h$ in $1+\mathbf{m} R$ such that $\phi_{h}$ is a split monomorphism. Now since $f$ is doubly monic, $R f+R h=R$. As $\phi_{f}$ is also a split monomorphism, $\phi$ is a split monomorphism. This completes the proof of (3.2).

(3.3) Lemma. Let $R=A\left[X, X^{-1}\right]$ be as above and $P, Q$ be two projective $R$-modules. Let $\phi, \phi^{\prime}: Q \rightarrow P$ and $\gamma: P \rightarrow Q$ be $R$-linear maps, such that $\gamma \phi^{\prime}=f \operatorname{Id}_{Q}$ for some doubly monic Laurent polynomial $f$. For integers $t>$ 0 , we write $\phi_{t}=\phi+\left(X+X^{-1}\right)^{t} \phi^{\prime}$. Then for large $t, \phi_{t}$ becomes a split monomorphism after inverting a doubly monic polynomial $f_{t}$.

Proof. As in [R], first we assume that $Q$ is free. We also have $\gamma \phi_{t}=\gamma \phi+$ $\left(X+X^{-1}\right)^{t} \gamma \phi^{\prime}=\gamma \phi+\left(X+X^{-1}\right)^{t} f \operatorname{Id}_{Q}$.

Since $Q$ is free, $\gamma \phi_{t}$ is a matrix. It is also clear that for large integers $t$, $\operatorname{det}\left(\gamma \phi_{t}\right)=f_{t}$ is a doubly monic Laurent polynomial. Therefore, $\phi_{t}$ becomes a split monomorphism after inverting $f_{t}$.

In the general case, find projective $R$-module $Q^{\prime}$ such that $Q \oplus Q^{\prime}$ is free. Define maps $G, G^{\prime}: Q \oplus Q^{\prime} \rightarrow P \oplus Q^{\prime}$ and $H: P \oplus Q^{\prime} \rightarrow Q \oplus Q^{\prime}$ by setting $G=\phi \oplus 0, G^{\prime}=\phi^{\prime} \oplus f \operatorname{Id}_{Q^{\prime}}$, and $H=\gamma \oplus \operatorname{Id}_{Q^{\prime}}$. It follows from above that for large $t, G_{t}$ becomes a split monomorphism after inverting a doubly monic 
polynomial $f_{t}$. Hence it also follows that $\phi_{t}$ becomes a split monomorphism after inverting $f_{t}$. This completes the proof of (3.3).

Now we are ready to prove our main theorem (2.1).

Proof of Theorem (2.1). Since $Q_{f}$ is isomorphic to a direct summand of $P_{f}$, we can find $R$-linear maps $\phi^{\prime}: Q \rightarrow P$ and $\gamma: P \rightarrow Q$ such that $\gamma \phi^{\prime}=f \operatorname{Id}_{Q}$ (possibly after replacing $f$ by a power of $f$ ).

Bar "-" will denote modulo $\mathbf{m}$, where $\mathbf{m}$ is the maximal ideal of $A$. So we have $\bar{\gamma} \bar{\phi}^{\prime}=\bar{f} \operatorname{Id}_{\bar{Q}}$. As $\bar{f}$ is doubly monic, $\bar{\phi}^{\prime}$ is a monomorphism.

Let $\operatorname{rank} P=r$ and $\operatorname{rank} Q=s$. We can find bases $\left\{\bar{p}_{1}, \bar{p}_{2}, \ldots, \bar{p}_{r}\right\}$ and $\left\{\bar{q}_{1}, \bar{q}_{2}, \ldots, \bar{q}_{s}\right\}$ for $\bar{P}$ and $\bar{Q}$, respectively, such that $\bar{\phi}^{\prime}\left(\bar{q}_{i}\right)=\bar{f}_{i} \bar{p}_{i}$ for some $f_{i}$ in $R$ and $i=1$ to $s$.

We define an $\bar{R}$-linear map $\bar{\phi}: \bar{Q} \rightarrow \bar{P}$ such that $\bar{\phi}\left(\bar{q}_{i}\right)=\bar{p}_{i+1}$ for $i=1$ to $s$ (note that $s<r$ ). For positive integer $t$, define $\bar{\phi}_{t}^{\prime}=\bar{\phi}+\left(X+X^{-1}\right)^{t} \bar{\phi}^{\prime}$. Since $\left\{\bar{p}_{1}, \bar{\phi}_{t}^{\prime}\left(\bar{q}_{1}\right), \ldots, \bar{\phi}_{t}^{\prime}\left(\bar{q}_{s}\right)\right\}$ is a part of basis for $\bar{P}, \bar{\phi}_{t}^{\prime}$ is a split monomorphism.

Let $\phi: Q \rightarrow P$ be a lift of $\bar{\phi}$ and $\phi_{t}^{\prime}=\phi+\left(X+X^{-1}\right)^{t} \phi^{\prime}$. Then $\phi_{t}^{\prime}$ is a lift of $\bar{\phi}_{t}^{\prime}$. By (3.3), for large $t \phi_{t}^{\prime}$ becomes a split monomorphism after inverting a doubly monic polynomial. Since $\bar{\phi}_{t}^{\prime}$ is also a split monomorphism, by (3.2) $\phi_{t}^{\prime}$ is a split monomorphism. So, $Q$ is a direct summand of $P$. This completes the proof of the theorem.

\section{ACKNOWLEDGMENT}

I thank Amit Roy for many useful discussions.

\section{REFERENCES}

[BR] S. M. Bhatwadekar and Amit Roy, Inversion of monic polynomial and existence of unimodular elements, Math. Z. 183 (1983), 87-94.

[NN] Budh Nashier and Warren Nichols, Ideals containing monics, Proc. Amer. Math. Soc. 99 (1987), 634-636.

[Q] D. Quillen, Projective modules over polynomial rings, Invent. Math. 36 (1976), 167-171.

[R] Amit Roy, Remarks on a result of Roitman, J. Indian Math. Soc. 44 (1980), 117-120.

[S] A. A. Suslin, Projective modules over polynomial rings are free, Soviet Math. Dokl. 17 (1976), 1160-1164. (English transl.) 